\title{
SPURIOUS LEAKY MODE SOLUTIONS AND EXPERIMENTAL VERIFICATION OF THE SECOND LEAKY WAVE
}

\author{
J. Zehentner, J. Macháč, J. Mrkvica, P. Vacek, J. Polívka* \\ Czech Technical University, Technická 2, 16627 Prague 6, Czech Republic \\ *Spacek Labs, Inc., 212 East Gutierrez Street, Santa Barbara, California 93101, USA
}

\begin{abstract}
This paper presents the revelation of spurious leaky mode solutions of the dispersion equation, and experimental confirmation of the occurrence of the second leaky wave on the slotline. The appearance of the spurious improper nonphysical solution instead of the true improper physical mode propagation constant is explained. A spontaneous jump of the dominant bound wave solution to an unwanted higher order bound wave solution is reported. Existence of the predicted second leaky surface wave excited on the slotline has been confirmed in two operation modes by two measuring methods.
\end{abstract}

\section{INTRODUCTION}

The harmfulness and usefulness of the leakage phenomenon has been demonstrated many times, e. g. in [1-5]. The spectral domain method has been adopted and is used currently for analysis of this phenomenon. Since it is an analytical method, nobody to our knowledge has reported any problem associated with a solution of the dispersion equation which provides us with leakage and phase constants. Consequently, it is generally thought that the procedure is clear, straightforward and results each time in single-valued solutions.

Detailed examination of this notorious process has led us to the revelation of its soft spot and has resulted in spurious, nonphysical solutions of the dispersion equation. This is a consequence of the lack of a sufficient number of constraints on the determinantal equation from which the propagation constant results. The same reason underlies the appearance of an additional spurious solution belonging to the higher order bound wave for which a higher number of basis functions is responsible.

The first experimental confirmation of the second leaky wave on conductor-backed coplanar strips appeared in [6]. We have experimentally verified the second leaky wave on the slotline already theoretically predicted in [7]. For this we combined the known field mapping techniques, utilizing a monochromatic signal with a new method based on noise signal measurement [8].
In this paper we report for the first time a spurious surface leaky wave solution of the dispersion equation and explain why and when it has to appear. On suitably designed slotlines we have confirmed the occurrence of the second leaky wave in the operation mode when the bound and the second leaky wave, on the one hand, and when the first and the second leaky wave, on the other hand, propagate simultaneously.

\section{SPURIOUS SOLUTIONS}

Shigesawa at al [9] reported for the first time a new improper real solution of the dispersion equation on conductor-backed coplanar strips and, in [10], also on the slotline. This solution helped to explain the evolution of the dispersion characteristics in dependence on the crosssectional dimensions of the line, and was treated as a new solution which completes the number of solutions below and above the spectral gap.

Let us have a look at this problem from another viewpoint in the case of the slotline. Let the propagation constant be $\gamma_{\mathrm{p}}=\alpha-\mathrm{j} \beta$, where $\alpha, \beta$ are the leakage and phase

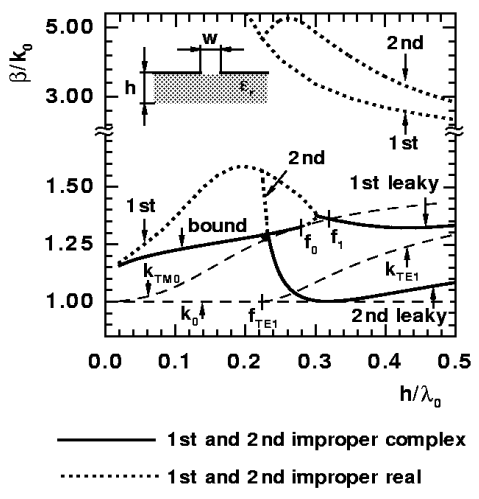

Fig. 1 Normalized $\beta / \mathrm{k}_{0}$ constant for the slotline with $w / h=0.4$ and $\varepsilon_{\mathrm{r}}=2.25$ as a function of normalized frequency $\mathrm{h} / \lambda_{0}$.

constant, respectively. Both of its components are obtained as a solution of the complex determinantal equation called the dispersion equation. Let $\mathrm{h} / \lambda_{0}=0.2$ and $\mathrm{h} / \lambda_{0}=0.29$ as in 
Fig. 1, and let us plot the determinant of the dispersion equation for the first leaky wave in dependence on $\beta / \mathrm{k}_{0}$, Fig. 2. It is seen from Fig. 2 that the determinant, the imaginary part of which is identically zero, crosses the axis twice and thrice, respectively. These intersection points correspond to the two and three improper real solutions $\left(\beta / k_{0}\right)$ in Fig.1. They had to occur due to the given profile of the real determinantal function.

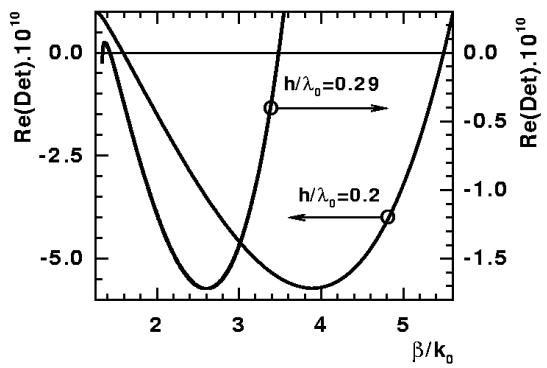

Fig. 2 Plot of the determinant belonging to the slotline specified in Fig. 1 when $h / \lambda_{0}=0.2$ and 0.29 in dependence on $\beta / \mathrm{k}_{0}$.
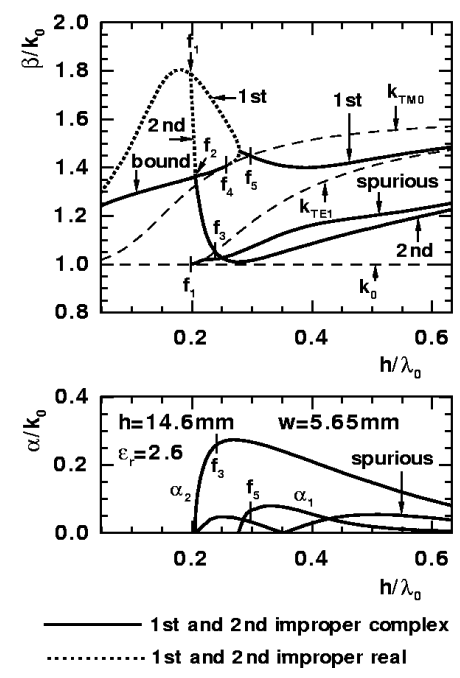

Fig. 3 The normalized phase $\beta / \mathrm{k}_{0}$ and leakage $\alpha / \mathrm{k}_{0}$ constants for the slotline with $\mathrm{w} / \mathrm{h}=0.387$ and $\varepsilon_{\mathrm{r}}=2.6$ as a function of normalized frequency $h / \lambda_{0}$.

Let us take another slotline with $\mathrm{w} / \mathrm{h}=0.387, \varepsilon_{\mathrm{r}}=2.6$ and draw its frequency dependent dispersion characteristics in Fig. 3. The relevant determinant is now complex. Its real part is shown in Fig $4 \mathrm{a}$, while its imaginary part within the same intervals $\alpha$ and $\beta$ is shown in Fig. 4b. Each surface crosses the zero plane in two curves. The solution of the determinantal equation represents the two points of intersection of these curves, as is seen in Fig. 5. The left root denoted 1 st is the propagation constant for the first leaky wave, while the right root, denoted $\mathrm{sp}$, is the spurious solution.

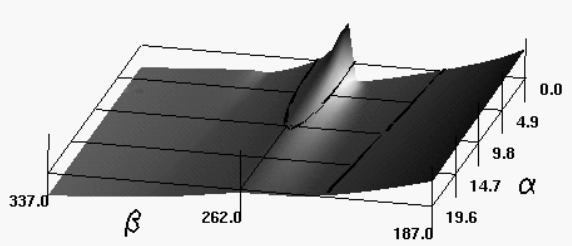

$\mathrm{a}$

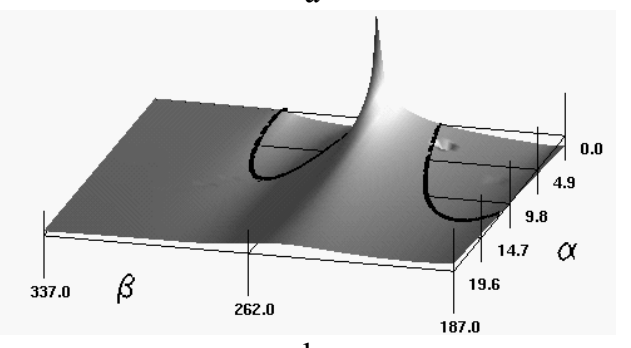

$\mathrm{b}$

Fig. 4 The real (a) and imaginary (b) part of the determinant for the slotline specified in Fig. 3 as a function of $\alpha$ and $\beta$.

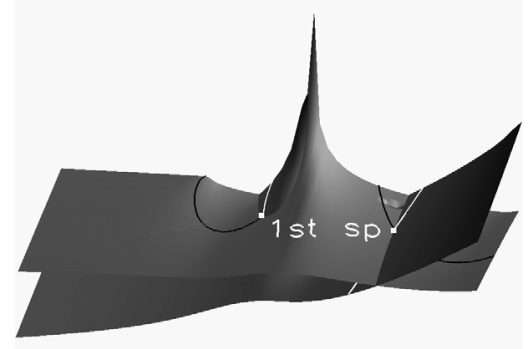

Fig. 5 Penetration of the surface of the real and imaginary part of the determinant given in Fig. 4 above the $\alpha, \beta$ plane.

Generally, all solutions of the dispersion equation are a straightforward consequence of the profile of the real and imaginary surface of the determinant. Mathematically, they are correct regardless of their physical meaning. We find already generally known and published solutions to be true, while the „new“ real solutions in [10], and also the complex solutions revealed now here are spurious. In any case, these spurious solutions are nonphysical, as follows from Figs. 1 and 3. Which solution we get depends on the starting point setting initializing the root-finder, i. e., on the ,valley“ into which the finder goes.

The better the basis functions describe reality, and the greater the number of basis functions that are accounted for, the more precise the results of the method of moments will be. Extensive tests have proved that, for the slotline, one basis function in the transversal direction and one in the longitudinal direction suffices in practice for calculation of the propagation constant. If we increase their number, e. g. for the bound wave, an interesting effect is observed. When the number of basis functions describing the strength of the electric field within the slot in the transversal direction exceeds the number of basis functions in the longitudinal direction by 2 , the root-finder provides the propagation constant of the higher order bound wave. 
This fact is not apparent at first glance, particularly when phase constant normalization is not applied. From the dominant bound wave viewpoint this solution is spurious, Fig. 6. This can be found out when we plot the field distribution within the slot calculated by means of the amplitudes of particular basis functions determined for an already known propagation constant, Fig. 7. It is seen that the root-finder applied to the dispersion equation produces a solution which is in fact valid for the mode higher than the examined one. The same effect was also observed on the coplanar waveguide.

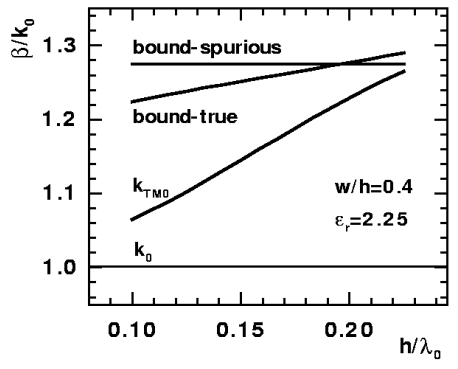

Fig. 6 True and spurious normalized propagation constant of the bound wave on the slotline with $\mathrm{w} / \mathrm{h}=0.4, \varepsilon_{\mathrm{r}}=2.25$. The number of basis functions in the longitudinal and transversal direction for the true solution is 1,1 and for the spurious solution it is 2,4 .

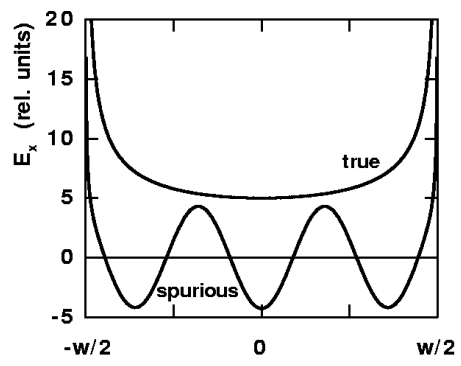

Fig. 7 Transversal electric field strength in the slot for a slotline with $\mathrm{w} / \mathrm{h}=0.4, \varepsilon_{\mathrm{r}}=2.25$ at $\mathrm{h} / \lambda_{0}=0.15$. The smooth curve results for one, the rippled curve for four basis functions.

\section{SECOND LEAKY WAVE MEASUREMENT}

Particular waves propagating on printed-circuit lines were detected by field mapping or by resonances when a monochromatic signal was applied [10]. In such a case, the measurement is done at a fixed frequency. Reflections of the monochromatic wave from any obstacles devalue field mapping, or even render it impossible. The whole field consists of many components depending on the frequency, e. g., direct radiation of the source, bound wave, one or more leaky waves, reflection from obstacles, one or more surface waves, etc. Field probing along the substrate surface requires a properly sized probe, exclusion of reflections, effective line feed and excitation of the desired wave. Some of these restrictive requirements can be avoided when active radiometry is applied [8].

However, practical measurement raised a number of questions. We therefore also applied a monochromatic signal in the already well known field mapping method, in order to compare it and combine it with the new active radiometric method.

To detect waves in the $\mathrm{X}$ band, three enlarged-scale slotlines were designed on a plexiglass, teflon and glass substrate with permittivity $\varepsilon_{\mathrm{r}}=2.6,2.08,6.3$, thickness $\mathrm{h}=14.6,4.4,4.0 \mathrm{~mm}$ and slotwidth $\mathrm{w}=5.65,3.3,1.2 \mathrm{~mm}$, respectively, Fig. 8 .

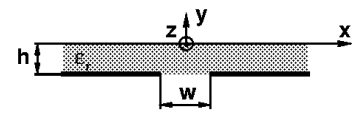

Fig. 8 Cross-section of a slotline

Simultaneous excitation of the 1st and 2nd leaky wave on the plexiglass slotline is identified in Fig. 9 where a monochromatic field probing record along the slot axis is shown. At 6.5 and $12 \mathrm{GHz}$, only the 1st and 2nd leaky wave occur, greatly attenuated, as is apparent from Fig. 3. The bound wave, weakly attenuated, produces a standing wave pattern at $3 \mathrm{GHz}$.

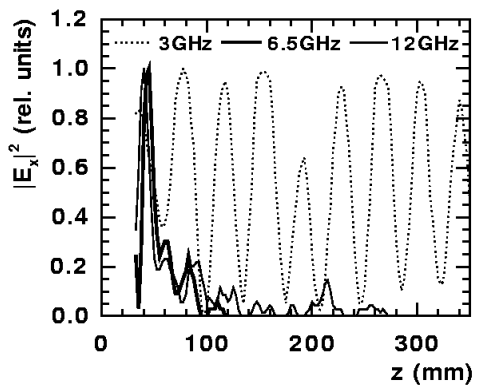

Fig. 9 Record of the $E_{x}$ field component along the plexiglass slotline taken at $3,6.5$ and $12 \mathrm{GHz}$

The $E_{x}$ field component record measured along the plexiglass slotline by the noise radiator is compared in Fig. 10 with the calculated superposition of the 1 st and 2 nd leaky waves in which their known propagation constants were applied. The ratio of these two wave amplitudes is $5 / 240$. Since the calculated curve fits the measured record well, we conclude that the 2nd leaky wave is launched dominantly and its leakage constant is checked properly.

Simultaneous propagation of the bound wave and the 2nd leaky wave was observed on the glass slotline. The dots in Fig. 11 mark the measured values of the normalized phase and leakage constants on the background of the calculated dispersion characteristics. 


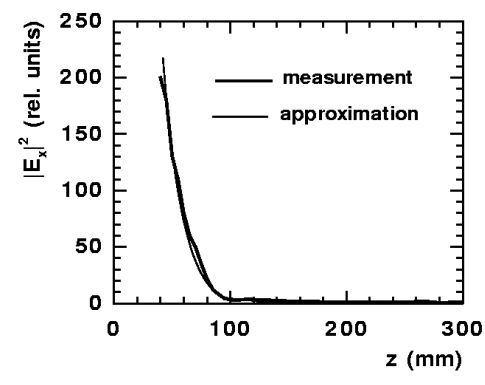

Fig. $10 \mathrm{E}_{\mathrm{x}}$ field component measured along the plexiglass slotline by the noise radiator at $10.75 \mathrm{GHz}$ and approximation of this record by two leaky waves.

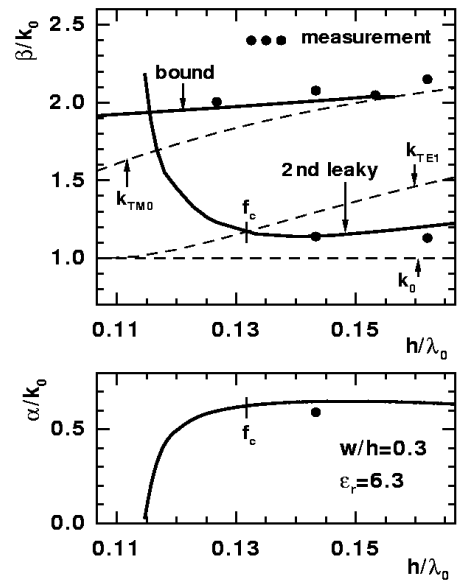

Fig. 11 Dispersion characteristics of a glass slotline with measured data when $\mathrm{w} / \mathrm{h}=0.3$ and $\varepsilon_{\mathrm{r}}=6.3$.

At $12.15 \mathrm{GHz}$ we detected the simultaneous presence of the 2 nd leaky wave and the surface $\mathrm{TM}_{0}$ wave. The leaky wave was quickly attenuated, while only losses in the substrate attenuated the surface wave. At $10.75 \mathrm{GHz}$ we found the 2nd leaky wave complex propagation constant and the phase constant of the bound wave. $\beta$ was determined from the distance of the resonance nodes, and $\alpha$ from comparison of the measured and calculated record of the fall of the $E_{x}$ field component along the line. At a frequency lower than $f_{c}$ only the bound wave was detected.

\section{CONCLUSIONS}

A warning about the danger of getting spurious solutions of the dispersion equation of printed-circuit lines has been given. This should be taken into account when the dispersion characteristics for a new line are computed for the first time and their complete picture is still unknown. Spurious solutions originate from an insufficient number of constraints put on the dispersion equation, as was shown for the slotline. Rugged profiles of the real and imaginary determinantal surfaces are responsible for this effect.
The 2nd leaky wave on the slotline was detected experimentally. Since the leaky wave is only a part of the whole excited field, this component is difficult to identify. We therefore designed slotlines reducing the possible wave spectrum. We observed the 2nd leaky wave propagating simultaneously with the bound or with the 1st leaky wave. A new active radiometric method, along with standard monochromatic techniques, was applied for the field mapping.

\section{ACKNOWLEDGMENT}

The work was done on the SP-2 computer at the Joint Supercomputer Center of the Czech Technical University, University of Chemical Technology and IBM Prague. Mr. $\mathrm{K}$. Hanton's assistance with 3D data visualization is gratefully acknowledged.

\section{REFERENCES}

[1] W. Menzel: A new travelling-wave antenna in microstrip. AEÜ, Band 33, 1979, April, pp. 137-140.

[2] A. A. Oliner: Scannable millimeter wave arrays. Polytechnic University, Weber Research Institute N. Y., Vol. 2, Chap. IX, Final Report POLY-WRI-1543-88, September 1988.

[3] H. Shigesawa, M. Tsuji: FDTD analysis of narrow pulse distortion on open and shielded coplanar waveguides. 23rd EuMC Conference Proceedings, Madrid, September 1993, pp. 281-284.

[4] P. Lampariello: New leaky-wave antennas for millimeter waves. Proceedings of International Symposium on Electromagnetic Theory, URSI, Thessaloniki, May 1998, pp. 479-481.

[5] T. Krems, A. Tessmann, W. H. Haydl, C. Schmelz, P. Heide: Avoiding cross talk and feed back effects in packaging coplanar millimeter-wave circuits. 1998 IEEE MTT-S IMS Digest, Vol. 2, Baltimore, June 1998, pp. 1091-1094.

[6] M. Tsuji, H. Shigesawa, H. Sannomiya, A. A. Oliner: The spectral gap when power leaks into more than one type of surface wave on printed-circuit lines. 1997 IEEE MTT-S IMS Digest, Vol. 2, Denver, June 1997, pp 483-486.

[7] J. Zehentner, J. Macháč, M. Migliozzi: Upper cut-off frequency of the bound wave and new leaky wave on the slotline. 1997 IEEE MTT-S IMS Digest, Vol. 2, Denver, June 1997, pp. 487-490.

[8] J. Zehentner, J. Macháč, J. Mrkvica, M. Sarnowski, J. Polívka: Field mapping by active radiometry. 1998 APMC'98 Proceedings, Vol. 1,Yokohama, December 1998, pp. 217-220.

[9] H. Shigesawa, M. Tsuji, A. A. Oliner: Evolution of new nonspectral real solution and its role in explaining the simultaneous propagation of both bound and leaky dominant modes on printed-circuit lines. Proceedings of the 1995 International Symposium on Electromagnetic Theory, URSI, St. Petersburg, May 1995, pp. 773-775.

[10] H. Shigesawa, M. Tsuji, A. A. Oliner: Simultaneous propagation of bound and leaky dominant modes on printedcircuit lines: A new general effect. IEEE Trans. Microwave Theory Tech., vol 43, 1995, 12, pp. 3007-3019. 